
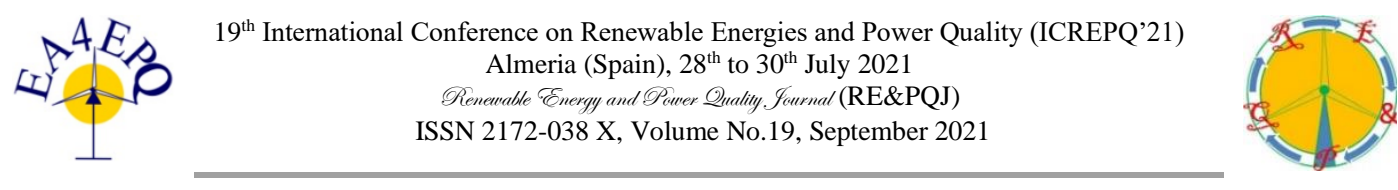

\title{
Heatsinks to Cool Batteries for Unmanned Aerial Vehicles
}

\author{
J. Galvão ${ }^{1,2}$, P. Faria ${ }^{3,5}$, A. Mateus ${ }^{4,5}$, T. Pereira ${ }^{4}$, S. Fernandes ${ }^{6}$ \\ ${ }^{1}$ Department of Electrical Engineering/ESTG, Polytechnic of Leiria, 2411-901 Leiria (Portugal) \\ Phone:+00351244820300, e-mail: jrgalvao@ipleiria.pt \\ ${ }^{2}$ R\&D Unit, Institute for Systems Engineering and Computers /INESCCoimbra (Portugal) \\ ${ }^{3}$ Departments of Mathematics/ESTG, Polytechnic of Leiria, 2411-901 Leiria (Portugal); paula.faria@ipleiria.pt \\ ${ }^{4}$ Department of Mechanical Engineering/ESTG, Polytechnic of Leiria, 2411-901 Leiria (Portugal); \\ artur.mateus@ipleiria.pt; 2182479@my.ipleiria.pt \\ ${ }^{5}$ Centre for Rapid and Sustainable Product Development (CDRSP) of the Polytechnic of Leiria (Portugal); \\ ${ }^{6}$ TEKEVER/Autonomous Systems, Rua das Minas 2, Zona Industrial2500-750 Caldas da Rainha (Portugal); \\ sofia.fernandes@tekever.pt
}

\begin{abstract}
This study aims to develop several different models of heatsinks, designed to cool a vertical take-off and landing unmanned aerial vehicle (UAV) battery, through topology optimization, aimed at being manufactured through selective laser melting (SLM) technology. A battery's temperature must be properly managed for a safe and efficient operation.

The methodology developed was with the support of software to carry out several simulations which, starting from several scenarios and restrictions imposed by the small space available to accommodate these small batteries in this type of aircraft. The conception resulted in several battery thermal management systems (BTMS) models, with different applications and efficiency degrees. A relevant aspect is the topology optimization being coupled to computational thermal analysis to reduce the mass of the heatsink whilst ensuring a maximum battery temperature threshold. Together with the use of topology optimization, the SLM process was selected to manufacture the heat sinks, under conditions of geometric freedom, using several high thermal conductivity metal alloys, such as, aluminium and copper to obtain the designed models.
\end{abstract}

Key words. BTMS, PCM, heatsink, physical model, topology optimization, battery.

\section{Introduction}

The airborne clean energy (ACE) system main purpose is to improve the cooling system for the batteries of a UAV, commonly referred to as a drone [1]. By improving the cooling capabilities of the battery thermal management system (BTMS) [2], it will allow a UAV to fly for longer and may even enable certain UAV designs to be fully electrically powered and in turn be more environmentally friendly. Improving the cooling capabilities of the UAV will require the knowledge of several subjects including BTMSs, how they work, what is the state of technology and where it can be improved to help reach the projects goal; battery technology and its state of the art; optimization strategies to improve the design of the batteries and its coolers; and additive manufacturing processes that will allow the testing of the developed designs and acquire its finals results.

\section{Technologies and Topology Analysis}

The current outline of the technologies related to the present work are related to BTMSs, the additive manufacturing technology of SLM and topology optimization, an advanced set of optimization methodologies that will aid in optimizing the battery cooling system that relates to this project.

\section{A. Technologies used: BTMS and SLM}

BTMSs are used along several applications of battery technologies available on the market. The SLM manufacturing technique will be used to manufacture a prototype of the cooling system for testing purposes.

Furthermore, a BTMS achieves its temperature goal by monitoring, controlling, and managing the battery system and its behaviour. Additional key features for a good BTMS are its ease of installation, compact size, rigidity, low cost and mass, its ease of maintenance and operation and the reliability of said system [3].

BTMSs can be divided into three categories, see Fig. 1:

$$
\begin{aligned}
& \text { - passive systems; } \\
& \text { - active systems; } \\
& \text { - hybrid systems. }
\end{aligned}
$$

for monitor, control and managing the temperature of a battery or battery pack to within a safe temperature range.

The passive systems are characterized for not requiring additional heating or cooling sources to heat/cool a system, while on the other hand active systems use an external energy source to improve its heating/cooling rate. Passive systems include passive air-cooling systems, passive liquid cooling systems, phase change material (PCM) based systems and heat-pipe based systems. General advantages of passive cooled systems are its low cost, high reliability, and ease of maintenance and/or operation, while its disadvantages are its lower performance if compared with active systems. This 


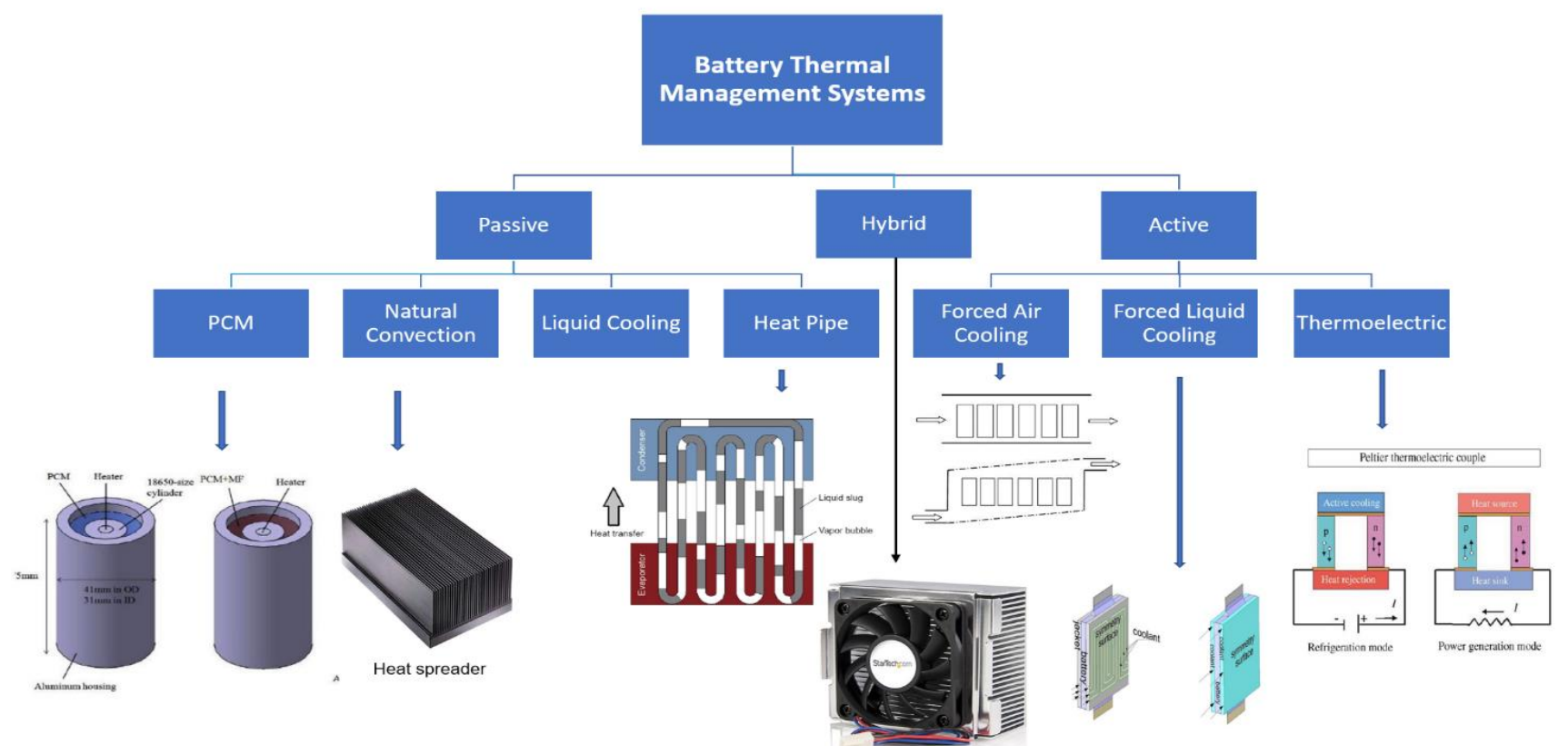

Fig. 1 - BTMS: battery thermal management several systems.

system include forced air cooling, forced liquid cooling systems as well as thermoelectric coolers. General advantages are the increased efficiency, the performance compared to passive systems, essential in critical applications, while its disadvantages are the increased price, maintenance requirements and the fact that it requires an external power source which might make them inviable for critically energy dense applications. Hybrid systems are systems that uses a combination of both passive and active heating/cooling methods [3].

\section{1) Passive cooling BTMSs, description:}

The safe temperature range for Lithium-Ion (Li-Ion) batteries is generally considered to be between $20^{\circ} \mathrm{C}$ and $40^{\circ} \mathrm{C}$, for optimal performance, and these temperatures should not exceed $60^{\circ} \mathrm{C}$. Failing to maintain the batteries between these temperatures will lead to premature failure, reduced longevity and performance and may in extreme cases lead to thermal runaway [2], [4]. Thermal runaway often happens due to exothermic reactions from poor charging, discharging or short circuit, these chemical reactions produce heat and gases. Thermal runaway causes large temperature rises, up to $500^{\circ} \mathrm{C}$, within the cell that are capable of permanently damaging the cell or the battery pack. Moreover, the increase in temperature within a cell, that happens during charge and discharge cycles, is non-uniform which must be taken into account during the development of a BTMS [5].

A passively air-cooled system is usually based on natural convection and may be assisted by using fins, either as heat spreader or as a heat absorber, and it is one of the simplest cooling methods. Due to its poor comparative performance this method is never used for critical applications, such as electric vehicle battery cooling systems or medium to high power electronics [3], [6], [7].

Passive liquid cooling systems use a liquid medium to transfer heat in or out of the system as shown in the rudimentary example by Pendergast et al. [8], where a triangular aluminium battery module is developed and then placed under water for cooling purposes.

PCM based thermal management systems use their capability of storing thermal energy as both sensible and latent heat, as well as being able to release that same energy in a reversible process. When the surroundings of a PCM increases in temperature it absorbs the heat and melts until the surrounding temperature goes bellow its phase changing temperature. The main advantage is the fact that it can store energy as latent heat at the working temperature of the system. The materials used are separated in organic, inorganic and eutectics materials with several subtypes, as seen in Fig. 2, based on their working temperature, from those, paraffin is one the most used PCM for BTMSs due to its low cost and its fitting phase change temperature.

It must be noted that accumulated heat should still be dissipated, especially once the PCM has fully melted [3], [7].

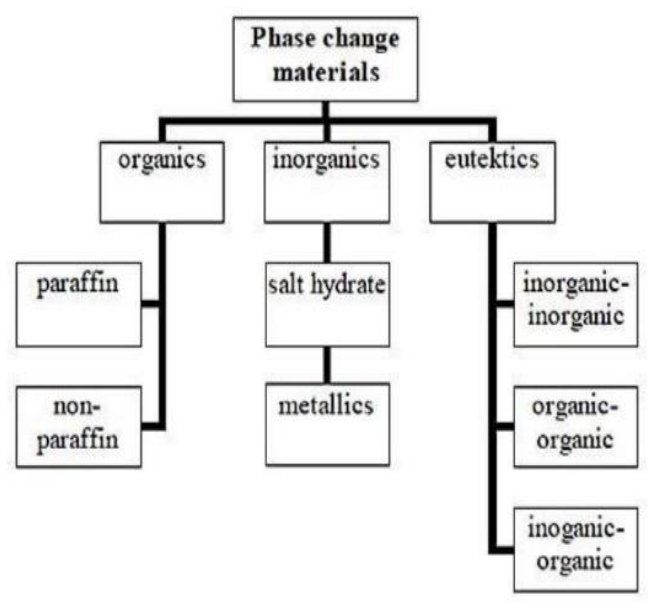

Fig. 2 - Phase change material types and subtypes. [9] 


\section{2) Active cooling BTMSs, description:}

Forced air cooling methods shares many similarities with its passive counterpart, but unlike its counterpart it uses either a fan or a blower to increase air flow. The addition of a fan/blower helps reducing maximum temperatures and with temperature uniformity, while still maintaining its simplicity and electrical safety of the overall system. Despite its improvements compared to passive air cooling it still fails to meet the required performance for certain applications due to the low heat capacity and conductivity of air [3], [5], [7].

Active liquid cooling methods use a pump or similar device, which causes these systems to be more complex, to promote flow of a liquid coolant used to remove the systems heat. The liquids, also known as heat transfer fluid (HTF), used have a higher specific heat capacity than air making them more efficient. The HFTs normally used include water, glycols, acetone, and oils. There are two types of active liquid cooling, direct contact, where the battery is in direct contact with the coolant, and indirect contact, where the battery is not in direct contact with the coolant.

Direct contact uses a dielectric fluid, usually mineral oil with or without the addition of nanoparticles, that improve its thermal properties, and can be more compact and have higher cooling capabilities than its counterpart. Nevertheless, sometimes direct cooling is not practical. Indirect cooling can be done with the use of a cold plate or a tube. Thera are three possible configurations for the cold plates, one where the cold plate channels are embedded into the battery monomer, another where it is placed in between the cells and a final one where it is in contact with the battery sides. There also two possible flow path configurations for the liquid in forced liquid cooling, parallel flow and serpentine flow. One advantage for indirect cooling is the fact that its HFTs have lower viscosity and require less energy to power the pump as a result [3], [5], [7].

A thermoelectric cooler (TEC) is a solid-state device that works based on the Peltier effect. The Peltier effect happens when a current goes through a thermoelectric material creating a temperature differential between the cold side, where heat is being absorbed, and the hot side/ heat sink, where the heat is channelled through the thermoelectric material and expelled. A thermoelectric couple is composed by a n-type and a p-type semiconductor material that are connected through metallic electrical contacts [3], [10].

\section{3) SLM manufacturing technique}

Another technology used is SLM. It is an additive manufacturing technology and as aforementioned it will be used to produce the prototype. This englobes every technology that uses computer aided design (CAD) data to produce parts or objects by depositing material directly into the work surface in a layer-by-layer fashion. This enables the manufacture of stronger and lighter parts with complex geometries in a multitude of materials be it polymers, ceramics or metals [11]. SLM was selected for its capabilities of manufacturing with metallic powders and the resulting properties of the parts manufactured. In Fig. 3 a part manufactured through SLM is seen having a complex internal lattice structure.
SLM uses a laser to selectively scan two-dimension sliced sections, of a tree-dimension CAD model, out of a powdered metal bed in a layer-by-layer fashion. The manufacturing process of a SLM part is as follows:

- Firstly, a layer of powdered metal is deposited on top of the base plate, followed by the laser scan of the first section where the metal is fully molten.

- Secondly, the building platform is lowered by the same amount of the layer thickness, followed by the deposition of a new layer of powdered metal.

- Lastly, the laser beam scans a new section in such a way that the molten powder consolidates with the previous bottom layer.

- The process is repeated until the final part is reached.

The main advantages of this process are its ability to produce parts with highly complex geometries and higher degrees of functionality, the shorter time to market of the developed products, the possibility for mass customization and the flexibility to work with a varied number of different materials, such as aluminium, copper, titanium and other metal alloys [12], [13].
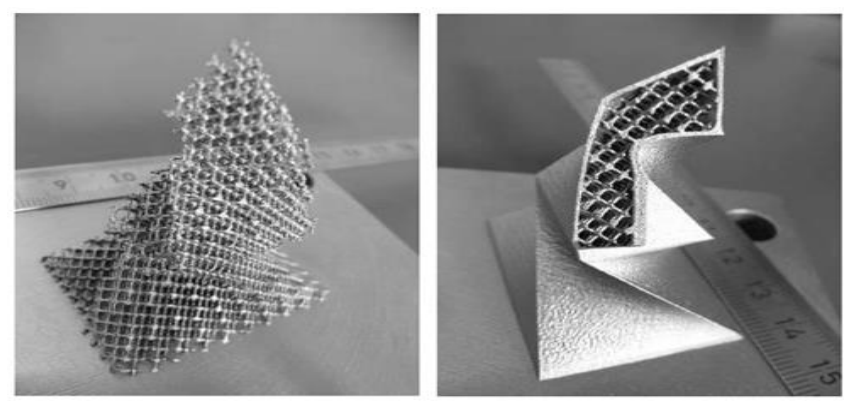

Fig. 3 - SLM part manufactured and inside a complex internal grid. [14]

\section{B. Topology Analysis and Optimization}

Topology optimization is the process that pertains to the calculation of the optimized layout and connectivity of voids within a certain design area. This allows for a greater degree of freedom of design compared to size and shape optimization, that deal with certain variables only, such as thickness or cross-section area of a feature, or with predetermined designs geometric features. Topology optimization can have great significance in early conceptual design stages since any change within the initial stages of design will notably alter the final performance. Many of the applications of topology optimization include single or a combination of structural, thermal, acoustic, fluid flow and material design applications as well as other multiphysics subjects. There are several methods for structural topology optimization, as shown in Fig. 4, being the following [14]:

- Density-based methods which are based on a "fixed domain of finite elements" where the goal is to minimize an objective function, usually compliance, by determining whether a certain element should be comprised of solid material or void. Further constraints are added such the amount of material that can be used.

- Hard-kill methods for topology optimization are based on the removal or addition of a finite amount of material from 
the design space. Such removal or addition is based on heuristic criteria that may or not be founded in sensitivity data and unlike density-based methods the discrete design space is not relaxed. The most popular of these methods is the Evolutionary Structural Optimization (ESO) method which has several variants including Bidirectional ESO (BESO), "soft-kill" BESO and genetic based ESO/BSO. One advantage of "hard-kill" methods is their simplicity to be utilized and added to commercial finite element packages.

- Boundary variation methods define structural boundaries based on implicit functions instead of explicit parameters. There are two boundary variation techniques the level set method and the phase-field method. Both methods produce results with smooth and clear edges that do not necessitate much post-processing. Unlike shape optimization techniques it allows for the formation, disappearance, and merger of voids besides the moving of the structural boundaries [14].

- And others developed methods inspired on cellular division.

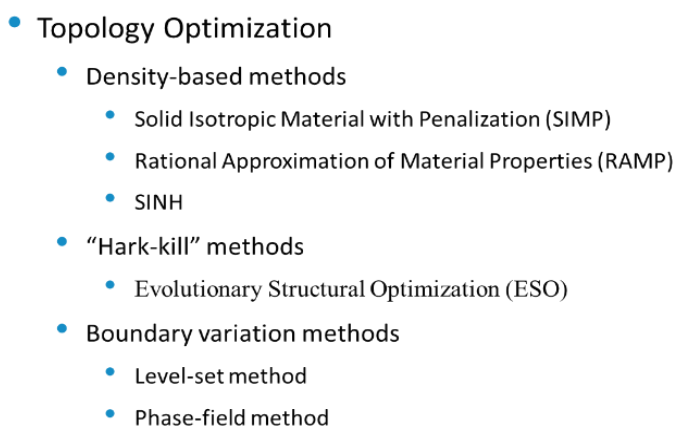

Fig. 4 - Topology optimization methods summary.

\section{Methodology}

The software used to support the development of the heatsink model to implement and optimize, which considers several solutions, scenarios and initial conditions is Ansys Workbench 2019 R3 [15]. The methodology consists of different parts described below:

a) Model Assessment

The assessment is comprised by the analysis of the battery's physical model, its framing within the UAV, as well as the properties of the materials considered for the heatsink.

The CAD model used for the optimization problem is divided into three distinct volumes, as follows:

- The battery, which is based on a $5000 \mathrm{mAh}$ (mili Ampere hour) lithium polymer battery from Gens Ace. It is parallelepiped in shape, $161 \times 46 \times 51 \mathrm{~mm}$ in size and has a mass of $808 \mathrm{~g}$, see Fig. 5. It has $63.7 \mathrm{~V}$ (Volt) lithium polymer cells in series with a total voltage of $22.2 \mathrm{~V}$ for the battery pack and it has a 60C (Coulomb) discharge rate [19]. The battery has an estimated heat output of $92.4 \mathrm{~W}$ and working temperature range of $+20^{\circ} \mathrm{C}$ to $+65^{\circ} \mathrm{C}$.

- A layer around the battery, named excluded region, see Fig. 5 , used to transfer the heat directly away from the battery through conduction as well as serving as support to which ever geometry is generated during the optimization step. This section has a uniform thickness around the battery, for the sake of simplicity, that could be controlled for to reduce and optimise the overall weight of the final geometry.

- The last volume pertains to the design volume, or design region, see Fig. 5, for the topology optimization. The original design volume is the remainder volume from the parachute bay not utilised by the battery and the excluded region. The design region was simplified into a parallelepiped from its original geometry to simplify the mesh throughout the development and optimization which will take place to solve the problem.

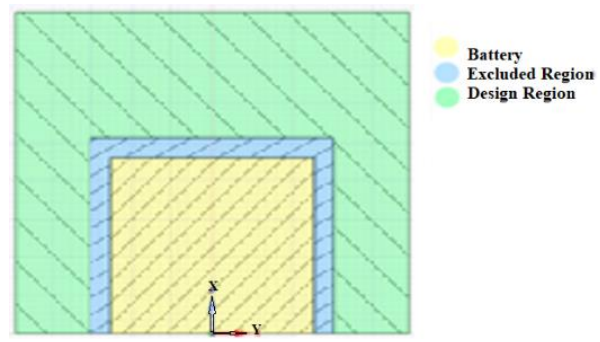

Fig. 5 - Battery and heatsink, excluded and design regions, volumes used for the development of the final model.

The materials used in the development of the problem include: a battery material simplified due to the complex construction of a lithium-ion battery pack; the values of the thermal conductivities; the specific heat of a cycled high voltage graphite/ $/ \mathrm{LiCoO}_{2}$ battery; dimensions and mass of the battery. The battery's density is $2.139 \mathrm{~g} / \mathrm{cm}^{3}$, its inplane and cross-plane thermal conductivity is $34.43 \mathrm{~W} / \mathrm{m} . \mathrm{K}$ and $1.39 \mathrm{~W} / \mathrm{m} . \mathrm{K}$ respectively, its specific heat is 1.028 $\mathrm{J} / \mathrm{g} .{ }^{\circ} \mathrm{C}$, and it has a volume of $377706.0 \mathrm{~mm}^{3}$. The materials considered for both the excluded and design regions are the following, seen in Fig. 6 [16], [17], [18].

\begin{tabular}{|c|c|c|}
\hline Material & Density $\left(\mathrm{g} / \mathrm{cm}^{3}\right)$ & Thermal conductivity $(\mathrm{W} / \mathrm{m} . \mathrm{K})$ \\
\hline Aluminium & 2.6989 & 210 \\
\hline AlSi $_{10} \mathrm{Mg}$ & 2.67 & $170 \pm 5$ (Heat treated) \\
\hline AlSi $_{7} \mathrm{Mg}_{0.6}$ & 2.67 & $180-190$ (Heat treated) \\
\hline Copper & 8.93 & 385 \\
\hline $\mathrm{CuNi}_{2} \mathrm{SiCr}$ & 8.85 & 190 (Precipitation hardening) \\
\hline
\end{tabular}

Fig. 6 - Material properties for the considered alloys.

\section{b) Workflow Development}

In this subsection, first and foremost, the topology optimization methodologies used by the analysis software will be described. Following, a brief explanation of the workflow of the problem and how it was setup in the used software will be done,

The workflow is separated in three sections are as follows: - A thermal analysis is first done to serve as an input and to model the thermal behaviour for the topology optimization step, here the geometry, in Fig. 5, is imported into the software, next boundary conditions and loads are defined, an internal heat generation load set on the battery, and as boundary conditions an initial temperature of $25^{\circ} \mathrm{C}$ and a convection heat transfer coefficient of $70 \mathrm{~W} / \mathrm{m}^{2}{ }^{\circ} \mathrm{C}$ due to a software limitation requiring the use of a steady-state thermal analysis instead of a transient thermal one. 
- The topology optimization step is where the geometry will be optimized according to a topology optimization algorithm. In this stage the design and excluded regions are setup as well as the optimization objective, in this case reducing the mass of the heatsink, and convergence criteria, a maximum temperature of $60^{\circ} \mathrm{C}$ on the battery.

- The verification step is used to assess the veracity of the results by importing and refining the optimized geometry followed by the assessment of its transient thermal behaviour, at a convection heat transfer coefficient of $5 \mathrm{~W} / \mathrm{m}^{2}{ }^{\circ} \mathrm{C}$ that will then be compared to the standalone battery's thermal behaviour.

\section{Results and Discussion}

In this subsection the results from the thermal analysis and topology optimization for the model using the $\mathrm{AlSi}_{10} \mathrm{Mg}$ alloy will be presented and briefly discussed.

The heat distribution within the battery at $t=315 \mathrm{~s}$ where it is visible that the hottest spot is found in the bottom of the battery, see Fig. 7. The maximum temperature of the battery model without heatsink over time is shown and it is possible to see that around $\mathrm{t}=141 \mathrm{~s}$ it reaches the $40^{\circ} \mathrm{C}$ and that around $\mathrm{t}=315 \mathrm{~s}$ it reaches the $60^{\circ} \mathrm{C}$ threshold at the constant heat load, see Fig. 8.

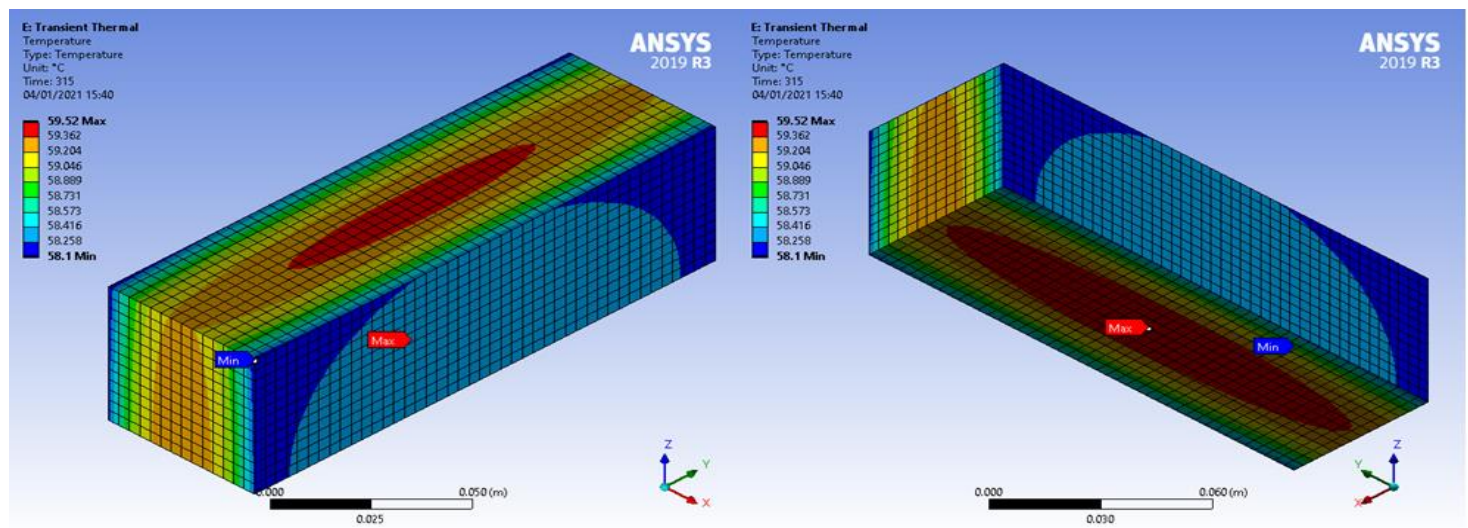

Fig. 7 - Battery heat distribution at $\mathrm{t}=315$ seconds.

According to the software's documentation, Ansys uses the SIMP penalization method a density-based method, for topology optimization using thermal loads as well as an external mechanical loading component.

Ansys uses the Sequential Convex Programming (SCP) approximation method to solve the optimization problem. SCP is an extension of the Method of Moving Asymptotes (MMA); a nonlinear approximation algorithm that approaches the solution of a topology optimization problem by solving a succession of convex separable subproblems. SCP requires the derivatives of all the functions of the topology optimization problem, ensuring convergence by dismissing steps which do not result in an optimal solution of the topology optimization problem [19], [20].

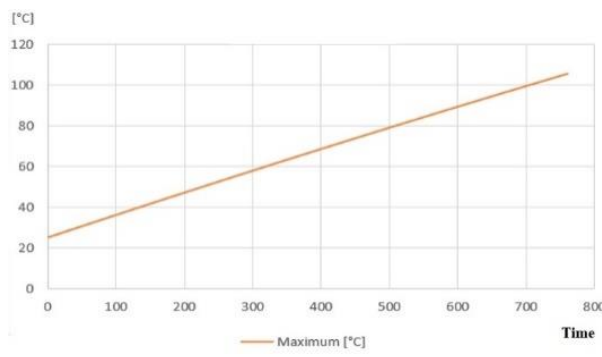

Fig. 8 - Battery's maximum temperature increase over time in the model without heatsink.

The assembly of heatsink different iterations during the topology optimization process and the optimized heatsink model with a mass of $728.6 \mathrm{~g}$ is shown in Fig. 9.

The next simulated case is to obtain the graph, Fig. 10, of the maximum battery's temperature over time with the heatsink and it reaches the $40^{\circ} \mathrm{C}$ threshold around $\mathrm{t}=199 \mathrm{~s}$ and it reaches the $60^{\circ} \mathrm{C}$ threshold around $\mathrm{t}=552 \mathrm{~s}$.

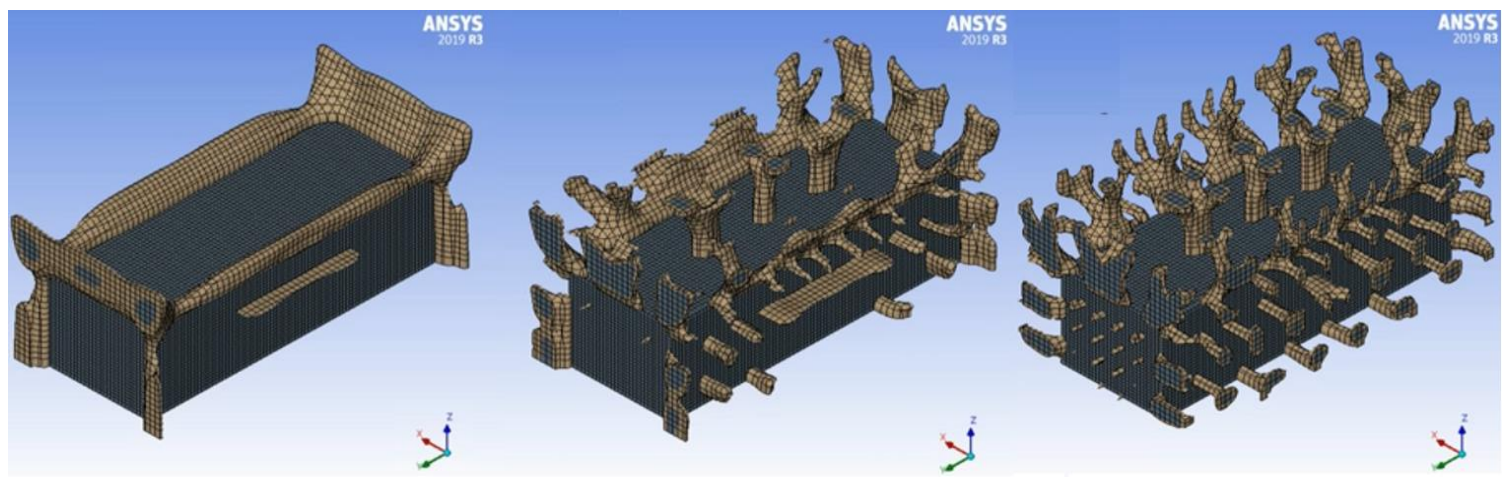

Fig. 9 - Assembly of heatsink different iterations. 
The heat distribution of the model at $t=552 \mathrm{~s}$ where the hottest spot is still at the bottom of the battery, see Fig. 11.

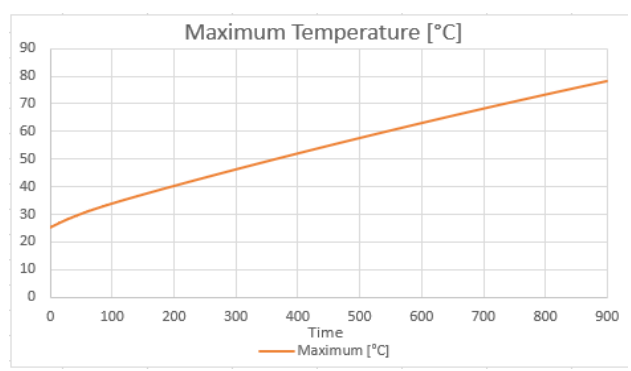

Fig. 10 - Battery's maximum temperature increase over time in the model with the heatsink.

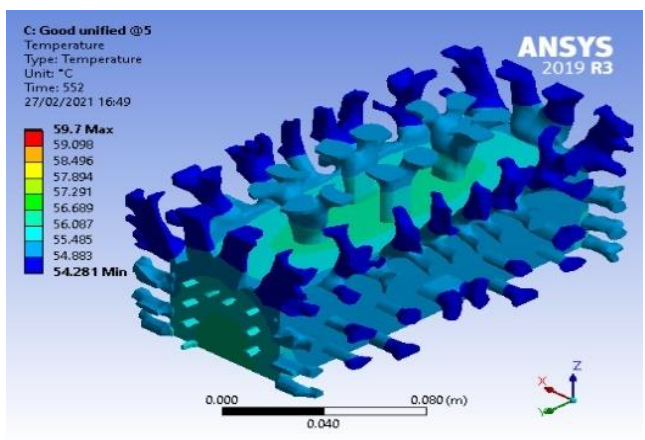

Fig. 11 - Heat distribution of the model at $\mathrm{t}=552$ seconds.

By comparing the evolution of the maximum temperature of the battery along time for both models it is evident the increase of $41.1 \%$ and $75.2 \%$ in the time necessary for the temperature to reach $40^{\circ} \mathrm{C}$ and $60^{\circ} \mathrm{C}$, respectively.

\section{Conclusion}

As evidenced by the a fore mentioned results there is an increase in the cooling performance of the BTMS and increase in the time required by the battery to reach its maximum temperature threshold.

This will in turn improve the longevity and safety of the battery. Its main limitations are the still limited cooling performance due to the poor cooling characteristics of air, as well as, the added weight of the heatsink that might be unwanted in critical applications.

Albeit further improvements could be achieved, for instance, by improving the heat transfer rate between the heatsink and the air or by individually cooling each cell through direct contact with heatsink.

\section{Acknowledgement}

This research was supported by the UIDB /00308/2020 project of the Science and Technology Foundation (FCT) of Portugal.

\section{References}

[1] ScienceDirect, "Unmanned Aerial Vehicles - an overview, ScienceDirect Topics", https://www.sciencedirect.com /topics/engineering/unmanned-aerial-vehicles (in Jan. 2021)

[2] R. Rizk, et.al., "Passive Cooling of High Capacity LithiumIon batteries," in 2018 IEEE - INTELEC Conference, 2018, pp. 1-4, doi: 10.1109/INTLEC.2018.8612368.

[3] A. R. M. Siddique, et. al., "A comprehensive review on a passive (phase change materials) and an active (thermoelectric cooler) battery thermal management system and their limitations," Journal of Power Sources. 2018, doi: 10.1016/j.jpowsour.2018.08.094.

[4] T. Reddy, Linden's Handbook of Batteries, 4th Edition. McGraw-Hill Education, 2010.

[5] L. Ianniciello, et. al., "Electric vehicles batteries thermal management systems employing phase change materials," Journal of Power Sources. 2018, doi: 10.1016/j.jpowsour.2017.12.071

[6] Z. Rao and S. Wang, "A review of power battery thermal energy management," Renew. Sustain. Energy Rev., vol. 15, no. 9, pp. 4554-4571, Dec. 2011, doi: 10.1016/J.RSER.2011.07.096.

[7] W. Wu, et.al.,"A critical review of battery thermal performance and liquid based battery thermal management," Energy Convers. Manag., vol. 182, pp. 262-281, 2019, doi: 10.1016/j.enconman.2018.12.051.

[8] D. R. Pendergast, et. al., "A rechargeable lithium-ion battery module for underwater use," J. Power Sources, vol. 196, no. 2, pp. 793-800, Jan. 2011, doi: 10.1016/J.JPOWSOUR.2010.06.071.

[9] S. Kalaiselvan, et al., "Solar PV Active and Passive Cooling Technologies - A Review," in 2018 Int. Conference on Comp. Power, Energy, Inf. and Com. (ICCPEIC), 2018, pp. 166-169, doi: 10.1109/ICCPEIC.2018.8525185.

[10] T. M. Tritt, "Thermoelectric Materials: Principles, Structure, Properties and Applications," Encycl. Mater. Sci. Technol., pp. 1-11, Jan. 2002, doi: 10.1016/B0-08043152-6/01822-2.

[11] "What is Additive Manufacturing? GE Additive." https://www.ge.com/additive/additive-manufacturing (accessed Feb. 29, 2020).

[12] "Selective Laser Melting Technology HRSflow." https://www.hrsflow.com/ww/en/solutions/processoptimization/slm (accessed Mar. 31, 2020).

[13] D.-A. Türk, et al., "Composites Part Production with Additive Manufacturing Technologies," Procedia CIRP, vol. 66, pp. 306-311, Jan. 2017, doi: 10.1016/J.PROCIR.2017.03.359.

[14] J. D. Deaton and R. V Grandhi, "A survey of structural and multidisciplinary continuum topology optimization: post 2000," Struct. Multidiscip. Optim., vol. 49, no. 1, pp. 1-38, 2014, doi: 10.1007/s00158-013-0956-z.

[15] Ansys, “Ansys Workbench 2019 R3." 2019/ https://support.ansys.com/portal/site/

[16] Gens Ace, "Gens Ace Li-Po 6S1P 5000mAh 60C Battery Packs with EC5 Plug - Gens Ace." https://www.gensace.de/gens-ace-5000mah-22-2v-60c6s1p-lipo-battery-pack.html\# (accessed Nov. 06, 2020).

[17] H. Maleki, et al., "Li-Ion polymer cells thermal property changes as a function of cycle-life," J. Power Sources, vol. 263, pp. 223-230, Oct. 2014, doi: 10.1016/j.jpowsour.2014.04.033.

[18] ZARE, "DMLS/SLM Aluminium AlSi10Mg," [Online]. https://www.zare.it/sites/default/files/manuals/DMLSSLM-Aluminium-AlSi10Mg_0.pdf (in Nov. 6, 2020)

[19] Ansys, "Topology Optimization Analysis." https://ansyshelp.ansys.com/account/secured?returnurl= /Views/Secured/corp/v195/wb_sim/ds_topology_optimi zation.html?q=topology optimization (in Dec. 28 , 2020).

[20] J. Deaton and R. Grandhi, "Stress-based Topology Optimization of Thermal Structures," 2013. 